\title{
NECESSARY AND SUFFICIENT CONDITIONS FOR THE REPRESENTATION OF A FUNCTION BY A WEIERSTRASS TRANSFORM
}

\author{
BY \\ D. V. WIDDER
}

Introduction. By the Weierstrass transform we mean the following special case of the convolution transform

$$
f(x)=c \int_{-\infty}^{\infty} e^{-(x-y)^{2} / 4} \phi(y) d y, \quad c=(4 \pi)^{-1 / 2},
$$

or more generally

$$
f(x)=c \int_{-\infty}^{\infty} e^{-(x-y)^{2} / 4} d \alpha(y) .
$$

Here, and throughout this paper, the Cauchy values of improper integrals are understood:

$$
\int_{-\infty}^{\infty}=\lim \int_{-S}^{R}
$$

where $R$ and $S$ tend to $+\infty$ independently. In (1), the function $\phi(y)$ is absolutely integrable, and in (2), $\alpha(y)$ is of bounded variation, both in every finite interval. We abbreviate (1) and (2) by $f(x)=W[\phi(x)]$ and $f(x)$ $=W_{1}[\alpha(x)]$, respectively. Our aim is to characterize the class of functions $f(x)$ which have the representation (2) with increasing $\alpha(y)$.

It is well known that Weierstrass used an integral of type (1) to give the first proof that a continuous function can be uniformly approximated by a polynomial. Hence the terminology. This name for the transform was used by Pollard $[1946 ; 317]\left({ }^{1}\right)$. Other names used in the literature are Gauss transform [Pollard 1943; 59], Gauss-Weierstrass transform [Hille 1948; 371] and Hille transform [González Domínguez 1941; 34]. Hille [1926] made an extensive study of the functions $f(x)$ and $\phi(x)$ of (1), referring to them as a "class of reciprocal functions."

A symbolic inversion of the transform (1) was first given by Eddington [1914] as follows:

Presented to the Society, April 28, 1951, under the title The Weierstrass transform of positive functions; received by the editors March 29, 1951.

(1) The dates in brackets refer to the bibliography at the end of this paper. A number following the date indicates the page of the article to which reference is made. 


$$
\phi(x)=e^{-D^{2}} f(x)=\sum_{k=0}^{\infty} \frac{(-1)^{k}}{k !} f^{(2 k)}(x) .
$$

Eddington was not concerned with questions of convergence, though he did observe that his attention had been called to the fact that the series (3) may well diverge for simple functions $f(x)$. It does so, in fact, for a large class of functions having the representation (1). Pollard [1946; 318] has shown that formula (3) may be given a meaning for all functions $f(x)$ $=W[\phi(x)]$, where $\phi(x) \in L^{2}(-\infty, \infty)$. This still excludes a large class of transforms (1). For example, $W\left[e^{x^{2} / 5}\right]=5^{1 / 2} e^{x^{2}}$. From our present point of view, this function must be included in the class considered. For, since $e^{x^{2} / 5}$ $>0, e^{x^{2}}$ has the form (2) with increasing $\alpha(y)$.

To see how the inversion (3) arises in a natural way, recall the Laplace transform

$$
e^{x^{2}}=c \int_{-\infty}^{\infty} e^{-x y} e^{-y^{2} / 4} d y, \quad-\infty<x<\infty .
$$

If we replace $x$ by the symbol $D$ (for differentiation) and interpret $e^{t D} \phi(x)$ in the usual way as $\phi(x+t)$, we obtain

$$
\begin{aligned}
e^{D^{2} \phi}(x) & =c \int_{-\infty}^{\infty} e^{-y^{2} / 4} e^{-y D} \phi(x) d y \\
& =c \int_{-\infty}^{\infty} e^{-y^{2} / 4} \phi(x-y) d y \\
& =W[\phi(x)]=f(x) .
\end{aligned}
$$

Thus we obtain (3) formally by performing the operation $e^{-D^{2}}$ upon both sides of (4). Our own interpretation of $e^{-D^{2}}$ may be deduced in a similar way from the known equation

$$
e^{-x^{2}}=c \int_{-\infty}^{\infty} e^{-y^{2} / 4} \cos x y d y, \quad-\infty<x<\infty .
$$

We are thus led to the definition

$$
\begin{gathered}
e^{-D^{2}} f(x)=c \int_{-\infty}^{\infty} e^{-y^{2} / 4}\{\cos y D\} f(x) d y, \\
\{\cos y D\} f(x)=\sum_{k=0}^{\infty}(-1)^{k} \frac{y^{2 k}}{(2 k) !} f^{(2 k)}(x) .
\end{gathered}
$$

It can be shown that this operator, suitably interpreted, inverts the integral (1) whenever it converges for $-\infty<x<\infty$. However, it is not the purpose of the present paper to prove this. We wish rather to use the operator (5) to 
guide us toward the characterization discussed above. Our result will be analogous to one of S. Bernstein [1928] giving necessary and sufficient conditions for the representation of $f(x)$ as a Laplace integral,

$$
f(x)=\int_{0}^{\infty} e^{-x y} d \alpha(y),
$$

with increasing $\alpha(y)$. These conditions may be predicted from the familiar inversion operator [Widder 1934] of (7):

$$
\left.\lim _{k \rightarrow \infty} \frac{(-1)^{k}}{k !} f^{(k)}(x) x^{k+1}\right|_{x=k / t}=\alpha^{\prime}(t) .
$$

Bernstein's characterization of the class of functions (7) is that they are all completely monotonic in a neighborhood of $+\infty$ :

$$
(-1)^{k} f^{(k)}(x) \geqq 0, \quad x_{0}<x<\infty, k=0,1,2, \cdots .
$$

That is, the inversion operator (8) (with limit sign omitted) is positive for all values of the parameter $k$. In a similar way we shall introduce a continuous parameter $t$ into our inversion operator and show that the desired class of functions $f(x)$ is essentially characterized by the condition

$$
e^{-t D^{2}} f(x) \geqq 0, \quad-\infty<x<\infty, 0<t<1 .
$$

We need to impose an additional condition here, but it is one that is satisfied by all functions (2), so that the conditions remain necessary and sufficient. We call attention to the papers of Pollard [1943; 59] and González Domínguez $[1941 ; 46]$ mentioned above, which give necessary and sufficient conditions of a different nature.

In the last section of the present paper it is shown how the flow of heat in a rod may be used as a guide to the results. Thus in (1) the function $f(x)$ is interpreted as the temperature of the rod one second after it was $\phi(x)$. Hence the inversion operator (5) must be a means of obtaining from the temperature $f(x)$ of the rod its temperature one second earlier. The chief tool in the proof of the sufficiency of the conditions (Theorem 3) is a theorem of the author [1944] concerning positive temperatures on an infinite rod. The main result of the present paper was announced earlier by the author [1951].

1. The operator $e^{-D^{2}}$. Eddington's definition of the operator $e^{-D^{2}}$, where the symbol $D$ indicates differentiation with respect to $x$, was

$$
e^{-D^{2}} f(x)=\sum_{k=0}^{\infty}(-1)^{k} \frac{f^{(2 k)}(x)}{k !} .
$$

However, this series may fail to converge for certain functions $f(x)$ in the class which we wish to consider. For example, if $f(x)=e^{x^{2}}$, series (1.1) becomes at $x=0$ 


$$
\sum_{k=0}^{\infty}(-1)^{k} \frac{(2 k) !}{k ! k !} .
$$

This series obviously diverges, is not even summable by Abel's method.

The definition of the operator given in equation (5) needs amplification. If taken to be applicable only when the series and integral both converge, its range is still too restricted. For example, one of the functions (2), the transform of a step-function with a single unit jump at the origin, is $e^{-x^{2} / 4}$. For this function

$$
\begin{aligned}
\{\cos y D\} f(x) & =\frac{f(x+i y)+f(x-i y)}{2} \\
& =e^{-\left(x^{2}-y^{2}\right) / 4} \cos \frac{x y}{2},
\end{aligned}
$$

and the integral (5) becomes

$$
c e^{-x^{2} / 4} \int_{-\infty}^{\infty} \cos (x y / 2) d y .
$$

This integral diverges for all $x$. When the integral (5) diverges we apply Abel summability. In this way we are led to the following formal definitions.

Definition 1. When $0<t<1$,

$$
e^{-t D^{2}} f(x)=\frac{c}{t^{1 / 2}} \int_{-\infty}^{\infty} e^{-y^{2} /(4 t)}\{\cos y D\} f(x) d y,
$$

where $\{\cos y D\} f(x)$ is defined by (5).

Definition 2.

$$
e^{-D^{2}} f(x)=\lim _{t \rightarrow 1-} e^{-t D^{2}} f(x) .
$$

It is clear that $e^{-D^{2}}$ can be applied, in accordance with these definitions, only to functions of class $C^{\infty}$. As a simple example take $f(x)=x^{2}$. Then

$$
\begin{aligned}
\{\cos y D\} x^{2} & =x^{2}-y^{2}, \\
e^{-D^{2}} x^{2} & =c \int_{-\infty}^{\infty} e^{-y^{2} / 4}\left(x^{2}-y^{2}\right) d y=x^{2}-2 .
\end{aligned}
$$

It will be convenient to have a relationship between the validity of the operations (1.1) and (1.3). Consequently, we prove:

Theorem 1. If the series (1.1) is summable, Abel, to the value $g(x)$, then $e^{-D^{2}} f(x)$ as defined in Definition 2 has the value $g(x)$.

For, let 


$$
g(x, t)=\sum_{k=0}^{\infty} a_{k} t^{k}, \quad a_{k}=\frac{(-1)^{k}}{k !} f^{(2 k)}(x),
$$

the series converging for $0<t<1$. Then by hypothesis $g(x)=g(x, 1-)$. We need show only that

$$
g(x, t)=\frac{c}{t^{1 / 2}} \int_{-\infty}^{\infty} e^{-y^{2} /(4 t)}\{\cos y D\} f(x) d y
$$

for $0<t<1$. This follows formally by an interchange of integral and summation signs

$$
\frac{c}{t^{1 / 2}} \int_{-\infty}^{\infty} e^{-y^{2} / 4 t} \sum_{k=0}^{\infty} \frac{k ! a_{k} \cdot y^{2 \cdot k}}{(2 k) !} d y=\sum_{k=0}^{\infty} a_{k} t^{k}
$$

since

$$
\frac{c}{t^{1 / 2}} \int_{-\infty}^{\infty} e^{-y^{2} / 4 t} y^{2 k} d y=\frac{(2 k) ! t^{k}}{k !} .
$$

To justify the interchange for a fixed $t, 0<t<1$, observe that by the assumed convergence of (1.4), $a_{k}=O\left(r^{-k}\right), k \rightarrow \infty$, for some value of $r, t<r<1$. If the integral (1.5) has a meaning when $a_{k}$ is replaced by its absolute value, or by $r^{-k}$, the validity of the interchange will be established. But the integral (1.5) is then seen to have the value

$$
\sum_{k=0}^{\infty} \frac{t^{k}}{r^{k}}=\frac{r}{r-t} .
$$

The theorem is proved, so that we may use equation (1.1) or (1.3) interchangeably if only we check the meaningfulness of the right-hand sides.

When the function $f(x)$ is entire another form of the operator $e^{-D^{2}} f(x)$ is usually more convenient to use. We prove:

THEOREM 2. If $f(x)$ is entire (real for real $x$ ), and if

$$
\lim _{t \rightarrow 1-} \frac{c}{t^{1 / 2}} \int_{-\infty}^{\infty} e^{-y^{2} / 4 t} f(x+i y) d y=g(x),
$$

then

$$
e^{-D^{2}} f(x)=g(x)
$$

By Taylor's theorem

$$
\begin{aligned}
f(x+i y) & =\sum_{k=0}^{\infty} f^{(k)}(x) \frac{(i y)^{k}}{k !}=e^{i y D} f(x) \\
& =\{\cos y D\} f(x)+i\{\sin y D\} f(x) .
\end{aligned}
$$


Our hypothesis contains implicitly the fact that the integral (1.6), and hence its real part and its imaginary part, converges for $0<t<1$. Since the imaginary part of $f(x+i y)$ is an odd function of $y$, the integral (1.6) clearly reduces to the integral (1.2), and our theorem is proved.

2. The representation theorem. The main result of the paper is contained in the following theorem.

THEOREM 3. For a function $f(x)$ to be represented in the form

$$
f(x)=c \int_{-\infty}^{\infty} e^{-(x-u)^{2} / 4} d \alpha(u), \quad c=(4 \pi)^{-1 / 2},
$$

where the integral converges for all $x$ and $\alpha(u)$ is nondecreasing, it is necessary and sufficient that

1. $f(x)$ should be entire;

2. $f(x+i y)=O\left(e^{y^{2} / 4}\right), y \rightarrow \pm \infty$, uniformly in $-R \leqq x \leqq R$ for every $R>0$;

3. $e^{-t D^{2}} f(x) \geqq 0,0<t<1,-\infty<x<\infty$.

As examples of functions satisfying these conditions we mention the following: $x^{2}+2, e^{x}, e^{x^{2}}, e^{-x^{2} / 4}$. The functions $\alpha(u)$ corresponding to the first three have the derivatives $u^{2}, e^{u-1}, 5^{-1 / 2} e^{u^{2} / 5}$, respectively. These derivatives are non-negative, as predicted by the theorem. The function $\alpha(u)$ corresponding to $e^{-x^{2} / 4}$ is clearly a step-function with a single jump of $c^{-1}$ at the origin.

3 . The necessity of the conditions. Let us assume that $f(x)$ has the representation (2.1). Then

$$
f(x)=c e^{-x^{2} / 4} \int_{-\infty}^{\infty} e^{x u / 2} e^{-u^{2} / 4} d \alpha(u),
$$

so that $f(x)$ is the product of an entire function by a bilateral Laplace transform which is convergent for all $x$. Thus $f(x)$ is entire. Moreover,

$$
|f(x+i y)| \leqq c e^{-x^{2} / 4} e^{y^{2} / 4} \int_{-\infty}^{\infty} e^{x u / 2} e^{-u^{2} / 4} d \alpha(u) .
$$

Hence condition 2 of the theorem is satisfied.

Finally, to prove condition 3, substitute the integral (2.1) in the integral (1.6),

$$
\frac{c^{2}}{t^{1 / 2}} \int_{-\infty}^{\infty} e^{-y^{2} / 4 t} d y \int_{-\infty}^{\infty} e^{-(x+i y-u)^{2} / 4} d \alpha(u),
$$

and interchange the two integrations to obtain formally

$$
\frac{c^{2}}{t^{1 / 2}} \int_{-\infty}^{\infty} e^{-(x-u)^{2} / 4} d \alpha(u) \int_{-\infty}^{\infty} e^{-i y(x-u) / 2} e^{-y^{2}(1-t) / 4 t} d y .
$$


Since the bilateral Laplace transform of $c e^{-t^{2} / 4 p}$ is $p^{1 / 2} e^{p x^{2}}$ for every positive constant $p$, this reduces to

$$
\frac{c}{(1-t)^{1 / 2}} \int_{-\infty}^{\infty} e^{-(x-u)^{2} / 4(1-t)} d \alpha(u) .
$$

This is seen to be non-negative, as desired. To justify the change in the order of integration we must replace the integrands in the iterated integral (3.1) by their absolute values and see if the resulting integral is convergent. Since $d \alpha(u) \geqq 0$, this amounts only to omission of the first factor in the inside integral. The resulting integral still converges and is easily evaluated to give for the iterated integral the value

$$
\frac{c}{(1-t)^{1 / 2}} \int_{-\infty}^{\infty} e^{-(x-u)^{2} / 4} d \alpha(u) .
$$

Since this integral converges for all $x$ by hypothesis, the interchange was justified, and the necessity of the conditions is established.

4. The sufficiency of the conditions. By conditions 1 and 2, which we are now assuming as hypotheses, the integral

$$
\frac{c}{t^{1 / 2}} \int_{-\infty}^{\infty} e^{-y^{2} / 4 t} f(x+i y) d y
$$

converges for all $x$ and for $0<t<1$ : As was shown in the proof of Theorem 2, this integral is equal to $e^{-t D^{2}} f(x)$ and is consequently greater than or equal to 0 by condition 3 .

Now form the function

$$
v(x, t)=e^{-(1-t) D^{2}} f(x)=\frac{c}{(1-t)^{1 / 2}} \int_{-\infty}^{\infty} e^{-y^{2} / 4(1-t)} f(x+i y) d y .
$$

It is well defined and greater than or equal to 0 for $-\infty<x<\infty, 0<t<1$.

By a change of variable, let us obtain an alternative expression for the integral (4.1). Let $a$ be an arbitrary constant. Since the function

$$
e^{(z-a)^{2} / 4(1-t)} f(z)
$$

is an entire function of $z=x+i y$, by Cauchy's theorem the integrals of this function along the two vertical lines $x=a$ and $x=0$ will be zero provided that

$$
\lim _{y \rightarrow \pm \infty} \int_{0}^{a} e^{(x+i y-a)^{2} / 4(1-t)} f(x+i y) d x=0 .
$$

But this is true by virtue of condition 2 . Hence

$$
\int_{-\infty}^{\infty} e^{-y^{2} / 4(1-t)} f(a+i y) d y=\int_{-\infty}^{\infty} e^{(i y-a)^{2} / 4(1-t)} f(i y) d y .
$$


Since $a$ was arbitrary we have for all $x$

$$
v(x, t)=\frac{c}{(1-t)^{1 / 2}} \int_{-\infty}^{\infty} e^{(i y-x)^{2} / 4(1-t)} f(i y) d y .
$$

It is now easy to show that $v(x, t)$ is a solution of the heat equation

$$
\frac{\partial^{2} v}{\partial x^{2}}=\frac{\partial v}{\partial t}, \quad-\infty<x<\infty, 0<t<1 .
$$

Formally,

$$
\begin{aligned}
\frac{\partial v}{\partial x}= & \frac{c}{(1-t)^{1 / 2}} \int_{-\infty}^{\infty} e^{(x-i y)^{2} / 4(1-t)} \frac{(x-i y)}{2(1-t)} f(i y) d y, \\
\frac{\partial^{2} v}{\partial x^{2}}= & \frac{c}{(1-t)^{1 / 2}} \int_{-\infty}^{\infty} e^{(x-i y)^{2} / 4(1-t)} \frac{(x-i y)^{2}}{4(1-t)^{2}} f(i y) d y \\
& +\frac{c}{(1-t)^{1 / 2}} \int_{-\infty}^{\infty} e^{(x-i y)^{2} / 4(1-t)} \frac{1}{2(1-t)} f(i y) d y, \\
\frac{\partial v}{\partial t}= & \frac{c}{2(1-t)^{3 / 2}} \int_{-\infty}^{\infty} e^{(x-i y)^{2} / 4(1-t)} f(i y) d y \\
& +\frac{c}{(1-t)^{1 / 2}} \int_{-\infty}^{\infty} e^{(x-i y)^{2} / 4(1-t)} \frac{(x-i y)^{2}}{4(1-t)^{2}} f(i y) d y .
\end{aligned}
$$

A comparison of these derivatives shows that equation (4.2) is satisfied. To justify the formal work it will be sufficient to show that the four integrals (4.3), (4.4) are uniformly convergent in the intervals $-R \leqq x \leqq R, \delta \leqq t \leqq 1-\delta$, where $R$ and $\delta$ are arbitrary positive constants $(R>1, \delta<1 / 2)$. By hypothesis 2 there is a positive constant $M$ such that each of the integrals in question is dominated, in the above intervals, by

$$
\frac{M c e^{R^{2} / 4 \delta}}{\delta^{5 / 2}} \int_{-\infty}^{\infty} e^{-y^{2} / 4(1-\delta)} e^{y^{2} / 4}\left(R^{2}+y^{2}\right) d y .
$$

Since this integral converges and is independent of $x$ and $t$, the desired uniform convergence is established. This proof also makes it evident that $v(x, t) \in C^{2}$ for $-\infty<x<\infty, 0<t<1$, so that $v(x, t)$ is a member of the class $H$ defined in $[1944 ; 87]$. Hence by Theorem 6 of that article, there exists a nondecreasing function $\alpha(u)$ such that

$$
v(x, t)=\frac{c}{t^{1 / 2}} \int_{-\infty}^{\infty} e^{-(x-u)^{2} / 4 t} d \alpha(u),
$$

the integral converging for $0<t<1,-\infty<x<\infty$.

By the definition of $v(x, t)$ as the integral (4.1) we have 


$$
v(x, t)=c \int_{-\infty}^{\infty} e^{-y^{2} / 4} f\left(x+i y(1-t)^{1 / 2}\right) d y .
$$

Since this integral is dominated in the intervals $-R \leqq x \leqq R, \delta \leqq t<1$ by the convergent integral

$$
c M \int_{-\infty}^{\infty} e^{-y^{2} / 4} e^{y^{2}(1-\delta) / 4} d y,
$$

we may apply Lebesgue's limit theorem to show that

$$
\lim _{t \rightarrow 1-} v(x, t)=f(x), \quad-\infty<x<\infty .
$$

We show next that the integral (4.5) converges when $t=1$. This follows from a familiar Tauberian-type argument. For every $x, t, R(0<t<1, R>0)$ we have

$$
\begin{aligned}
\frac{t^{1 / 2}}{c} v(x, t) & =\int_{-\infty}^{\infty} e^{-(x-u)^{2} / 4 t} d \alpha(u) \geqq \int_{x-R}^{x+R} e^{-(x-u)^{2} / 4 t} d \alpha(u) \\
& \geqq e^{-R^{2}(1-t) / 4 t} \int_{-R}^{R} e^{-u^{2} / 4} d \alpha(u+x) .
\end{aligned}
$$

Now let $t \rightarrow 1$ in this relation and use (4.6) to obtain

$$
c \int_{-R}^{R} e^{-u^{2} / 4} d \alpha(u+x) \leqq f(x) .
$$

Since $\alpha(u)$ is nondecreasing, this shows that (4.5) converges at $t=1$.

Finally, by an Abelian argument, the limit of the integral (4.5) as $t \rightarrow 1-$ is its value at $t=1$. To avoid repetition of this familiar argument, we may write the integral (4.5) as the sum of two others, corresponding to the intervals $(-\infty, 0)$ and $(0, \infty)$, and make the change of variable $u-x=v^{1 / 2}$, thus reducing each to a unilateral Laplace transform for which the appropriate Abelian theorem may be quoted [Widder 1946; 56].

If then we allow $t$ to approach 1 in (4.5), we obtain

$$
f(x)=c \int_{-\infty}^{\infty} e^{-(x-u)^{2} / 4} d \alpha(u), \quad-\infty<x<\infty,
$$

the desired result.

5. Physical interpretation. By a physical interpretation of the integrals entering into the above analysis we may see why the operator $e^{-D^{2}}$ should serve to invert the Weierstrass transform. It is well known that the integral

$$
\frac{c}{t^{1 / 2}} \int_{-\infty}^{\infty} e^{-(x-u)^{2} / 4 t} \phi(u) d u
$$


represents the temperature of an infinite rod at a point $x$ of the rod $t$ seconds after it was $\phi(x)$. Hence the function $f(x)=W[\phi(x)]$ gives the temperature of the rod one second after it was $\phi(x)$. The function

$$
v(x, t)=e^{-(1-t) D^{2}} f(x)
$$

was also seen to satisfy the heat equation and to approach $f(x)$ as $t \rightarrow 1-$. Consequently it represents the temperature of the rod $(1-t)$ seconds before it was $f(x)$. It is thus clear physically why the operation

$$
\lim _{t \rightarrow 0+} v(x, t)=\lim _{t \rightarrow 1-} e^{-t D^{2}} f(x)=e^{-D^{2}} f(x)
$$

should give the temperature, $\phi(x)$, of the bar one second before it was $f(x)$ and should thus invert the Weierstrass transform.

\section{BIBLIOGRAPHY}

1. S. Bernstein, Sur les fonctions absolument monotones, Acta Math. vol. 51 (1928) pp. 1-66.

2. A. Eddington, On a formula for correcting statistics for the effects of a known probable error of observation, Monthly Notices Royal Astronomical Society vol. 73 (1914) pp. 359-360.

3. A. González Domínguez, Contribución a la teoría de las funciones de Hille, Ciencia y Técnica no. 475 (1941) pp. 475-521.

4. E. Hille, $A$ class of reciprocal functions, Ann. of Ma,th. vol. 27 (1926) pp. 427-464.

5. - Functional analysis and semi-groups, Amer. Math. Soc. Colloquium Publications, vol. 31, 1948, pp. 1-528. $59-65$.

6. H. Pollard, Representation as a Gaussian integral, Duke Math. J. vol. 10 (1943) pp.

7. — Integral transforms, Duke Math. J. vol. 13 (1946) pp. 307-330.

8. D. V. Widder, The inversion of the Laplace integral and the related moment problem, Trans. Amer. Math. Soc. vol. 36 (1934) pp. 107-200.

9. - - Positive temperatures on an infinite rod, Trans. Amer. Math. Soc. vol. 55 (1944) pp. $85-95$.

10. - The Laplace transform, Princeton, 1946, pp. 1-406.

11. - Weierstrass transforms of positive functions, Proc. Nat. Acad. Sci. U S.A. vol. 37 (1951) pp. 315-317.

HARVARD UNIVERSITY,

Cambridge, Mass. 